PAEDIATRICS

\section{DIPSTICK ADEQUATE}

FOR FEBRILE UTI TEST

Urine dipstick alone (without urine microscopy) is an adequate screening test for febrile infants aged 1-90 days with suspected UTI, according to a recent study from researchers in Salt Lake City, Utah, USA. In this study, Glissmeyer et al. assessed data for 6,394 febrile infants who had been tested with urine dipstick, microscopy, and culture between 2004 and $2011,12 \%$ of whom were found to have a UTI.

Although combined urinalysis (dipstick plus microscopy) resulted in a slightly higher negative predictive value than dipstick alone (99.2\% versus $98.7 \%$ ), the positive predictive value was significantly lower using this approach $(51.2 \%$ versus $66.8 \%)$. The investigators calculated that eight febrile infants would be falsely diagnosed with a UTI using combined urinalysis for every one infant with a UTI who was initially missed by dipstick screening. In other words, for a group of 1,000 febrile infants, the addition of urine microscopy to dipstick testing would correctly identify five additional UTIs but falsely predict UTI in 41 infants compared with dipstick testing alone.

Importantly, patients with a UTI and negative dipstick test are unlikely to experience adverse outcomes while waiting for culture results, whereas false-positive results could lead to unnecessary interventions, including hospital admission. Furthermore, urine dipstick is a cheap and rapid screening tool that can be performed in an office setting without specialist training; microscopy, on the other hand, must be performed by technicians with specialist training using methods certified by the Clinical Laboratory Improvement Amendment.

"These data support the use of fewer screening tests for UTI in febrile infants while culture is pending," says Eric Glissmeyer, lead researcher of the study. "Performing additional screening tests beyond dipstick (like microscopy) would pick up a few more cases by increasing overall sensitivity but with the downside of leading to unneeded testing and unnecessary admissions. With dipstick plus microscopy, a positive test is only really true about half of the time, but when dipstick alone is performed, a positive test is really true more than two-thirds of the time."

Melanie Clyne

Original article Glissmeyer, E. W. et al. Dipstick screening for urinary tract infection in febrile infants. Pediatrics doi:10.1542/peds.2013-3291 